Quim. Nova, Vol. 35, No. 6, 1194-1197, 2012

\title{
ESTUDIO DEL COEFICIENTE DE REPARTO DE ÁCIDOS CARBOXÍLICOS GRASOS EN SISTEMAS MODIFICADOS HEPTANO/AGUA
}

\author{
Bélgica Bravo*, Betzhy Cárdenas, Gerson Chávez, Nacarid Delgado, Ana Cáceres, Fredy Ysambertt y Nelson Márquez \\ Departamento de Química, Facultad de Ciencias, Universidad del Zulia, 4001 Maracaibo - Zulia, Venezuela
}

Recebido em 9/11/11; aceito em 31/1/12; publicado na web em 30/4/12

\begin{abstract}
STUDY OF THE PARTITION COEFFICIENT OF FATTY CARBOXYLIC ACIDS IN MODIFIED HEPTANE/WATER SYSTEMS. The use of fatty acids (FAs) as amphiphiles is very important because they have a behaving similar to surfactants. The formulation for the preferential partition of these species was studied by varying the amount of salt at constant acid concentration. As the salt concentration increases, a Winsor I $\rightarrow$ III $\rightarrow$ II transition is observed for all the systems studied. Furthermore, the electrolyte concentration required to obtain the optimum formulation varies inversely with the chain length of the acid. The partition coefficient of the surfactant allows one to obtain thermodynamic information on the acid transfer process between the phases of the system.
\end{abstract}

Keywords: fatty acids; formulation variables; partition coefficient.

\section{INTRODUCCIÓN}

En la actualidad el empleo de sustancias anfifilicas juega un papel fundamental en diversos procesos industriales, como por ejemplo en la recuperación del petróleo, en la formulación de espumas, en estudios de estabilidad de diferentes emulsiones y microemulsiones entre otros, ${ }^{1,2}$ en este sentido los ácidos carboxílicos grasos (ACG) naturales, así como los sintéticos, son una fuente importante de materia prima que pueden emplearse como surfactantes. ${ }^{3}$ La capacidad de éstos ácidos para actuar como surfactantes y estabilizar emulsiones depende de su capacidad para adsorberse en una interfase, reducir en gran medida la tensión interfacial y formar una película adhesiva. ${ }^{4}$ La doble compatibilidad de estos compuestos les otorga ciertas propiedades muy interesantes y bastante útiles; en primer lugar, la ubicación preferencial en las superficies de los líquidos o en las interfases de compuestos no compatibles, por lo que sirven de puente entre ellas y facilitan su dispersión. En segundo lugar, la ubicación de las moléculas hace que disminuya la energía de superficie del sistema, modificando de esta forma la tensión interfacial en sistemas conformados por líquidos inmiscibles. ${ }^{5,6}$

En un sistema agua-hidrocarburo puede ocurrir un reparto de las especies anfifílicas entre las fases del sistema, por lo que es importante su determinación y cuantificación en cada fase; ya que, estas especies son las responsables de la disminución de la tensión interfacial. .,3,5,7-13 $^{2}$ Este reparto preferencial es afectado por las variables de formulación en el sistema (electrolitos, alcoholes, etc.), por lo que en este trabajo se evaluó el efecto que causa la adición de diferentes electrolitos en el coeficiente de reparto de los ACG.

\section{PARTE EXPERIMENTAL}

\section{Equipos}

Para la cuantificación del ACG en cada fase se empleó un cromatógrafo líquido de alta eficiencia (CLAE) tipo modular marca Waters, conformado por: un inyector universal U6K; bomba modelo 510, detector UV-VIS modelo 484 de longitud de onda variable, una interfase PE-Nelson modelo 900 series marca Perkin Elmer y un computador

\footnotetext{
*e-mail: belgicabravo@gmail.com
}

PC 5500 con software Turbochrome para el procesamiento de los datos. Se empleó una columna de fase reversa, con fase estacionaria de octadecilsilano (C18) marca Merck tamaño de partícula de $5 \mathrm{~mm}$ y dimensiones de $250 \mathrm{~mm}$ de longitud x 4,6 $\mathrm{mm}$ de diámetro interno, para el calentamiento de la columna se empleó una manta de calentamiento.

\section{Reactivos}

Para la preparación de los barridos de formulación se empleó como fase orgánica heptano (J. T. Beaker, grado analítico); como anfifílos los ACG: ácido dodecanóico (C12), ácido tetradecanóico (C14) y ácido hexadecanóico (C16) (Merck) y como co-surfactante se usó $n$-butanol (Scharlau 99,7\%). La fase acuosa estuvo constituida por: agua destilada, hidróxido de sodio (Merck, 98,9\%) y la sal en estudio; las cuales fueron: cloruro de sodio $\mathrm{NaCl}$ (99,5\%, Merck), nitrato de sodio $\mathrm{NaNO}_{3}\left(99,5 \%\right.$, Merck), sulfato de sodio $\mathrm{Na}_{2} \mathrm{SO}_{4}$ (99\%, Riedel de Haën), carbonato de sodio $\mathrm{Na}_{2} \mathrm{CO}_{3}(99 \%$, Riedel de Haën), fosfato ácido de sodio $\mathrm{Na}_{2} \mathrm{HPO}_{4}(99,9 \%$, Riedel de Haën). Se emplearon tubos de ensayo graduados y material de vidrio en general.

\section{Procedimiento}

Efecto de diferentes sales inorgánicas en el comportamiento de fases de sistemas ACG/n-heptano/agua/n-butanol

Se realizaron barridos de concentración de diferentes sales sódicas ( $\mathrm{NaCl}, \mathrm{NaNO}_{3}, \mathrm{Na}_{2} \mathrm{CO}_{3}, \mathrm{Na}_{2} \mathrm{SO}_{4}, \mathrm{Na}_{2} \mathrm{HPO}_{4}$ ) en sistemas ACG /agua/ heptano/n-butanol; mezclando en tubos de ensayo graduados (relación heptano/agua igual a uno), la fase acuosa $(\mathrm{NaOH} 2,4 \% \mathrm{~m} / \mathrm{v}$ y la sal en estudio) y la fase orgánica ( $n$-heptano con los ACG disueltos a una concentración de 0,2; 0,5 y 1,0\% m/v). Luego se adicionó el $n$-butanol al sistema hasta mantener una concentración constante del $3 \% \mathrm{v} / \mathrm{v} .{ }^{10}$ Los sistemas se agitaron suavemente cada dos h durante $12 \mathrm{~h}$, y luego se dejaron estabilizar por una semana a una temperatura de $25^{\circ} \mathrm{C}$. Luego se midieron los volúmenes de fase respectivos para realizar el cálculo del parámetro de solubilización, el cual es la relación entre el volumen de fase $\left(V_{0}\right.$ y $\left.V_{\mathrm{A}}\right)$ y la concentración en masa (Vs) del surfactante en el sistema:

$$
P S o=\frac{V o}{V s} \quad \text { y } \quad P S w=\frac{V_{A}}{V s}
$$


De acuerdo a las ecuaciones anteriores la formulación óptima, definida como PS* ${ }^{*}$ corresponde esencialmente al punto donde las dos curvas que indican la variación de los parámetros de solubilización en función de la variable en estudio se interceptan, esto es:

$$
P S o=P S_{A}=P S^{*}
$$

\section{Estudio del coeficiente de reparto de ACG en sistemas Winsor III} tendientes a formulación óptima

La fase acuosa, media (microemulsión) y orgánica de los sistemas Winsor III se separaron empleando una jeringa con una aguja de 30 $\mathrm{cm}$ de longitud, previendo la contaminación de las mismas; posteriormente el solvente se eliminó por evaporación en una estufa a 60 ${ }^{\circ} \mathrm{C}$ durante $42 \mathrm{~h}$. El producto seco de la fase orgánica, se disolvió en $\mathrm{MeOH}$ para el análisis por CLAE, mientras que las fases acuosa y media fueron disueltas en metanol, sonicadas durante 20 min y estas soluciones fueron filtradas mediante un papel Whatman $\mathrm{N}^{\circ} 40$ para separar la sal presente, y finalmente fueron aforadas a un volumen de $5 \mathrm{~mL}$ e inyectadas al cromatógrafo. El cálculo del coeficiente de reparto $\left(\mathrm{K}_{\mathrm{R}}\right)$ se realizó de la siguiente manera: una vez cuantificado el ACG presente en los sistemas Winsor III, se obtuvo la relación de la concentración de éste en la fase acuosa $\left([\mathrm{ACG}]_{\mathrm{A}}\right)$ respecto a la concentración presente en la fase orgánica ([ACG $]_{\mathrm{O}}$ ) empleando la Ecuación 3, a fin de relacionar el coeficiente de reparto de los ACG con la concentración y naturaleza del anión utilizado.

$$
K_{R}=\frac{[\mathrm{ACG}]_{O}}{[\mathrm{ACG}]_{A}}
$$

\section{RESULTADOS Y DISCUSION}

\section{Efecto de diferentes sales inorgánicas en el comportamiento de fases de sistemas ACG/n-heptano/agua/n-butanol}

La realización de barridos de formulación tiene como finalidad la determinación de un sistema tendiente a formulación óptima, para ello se evaluó la influencia de la concentración y la naturaleza de diferentes electrolitos en la fase acuosa de sistemas ACG /n-heptano/agua/ $n$-butanol. Al modificar la salinidad de la fase acuosa se modifica la afinidad del surfactante por esta fase y por lo tanto se modifica el reparto preferencial de estas especies anfifílicas entre las fases del sistema.

La conducta de fase de los sistemas surfactante/aceite/agua usualmente es explicada empleando el modelo hipotético cualitativo de Winsor. ${ }^{14}$ Estos sistemas se pueden clasificar en cuatro tipos, conocidos como Winsor tipo I, tipo II, tipo III y las microemulsiones tipo IV. En los sistemas Winsor tipo I, ó aceite en agua, la microemulsión contiene un exceso de fase orgánica y micelas de surfactante en la fase microemulsión. En la fase microemulsión, la solubilidad del aceite está dada por la partición del surfactante en micelas dispersas en la fase continua acuosa. Para el sistema Winsor II ó agua en la microemulsión orgánica, un exceso de la fase acuosa está en equilibrio con la fase microemulsión. En este caso, el agua está solubilizada en las micelas inversas, dispersas en la fase continua orgánica. Ajustando las variables de formulación (salinidad, temperatura, entre otras), se obtiene un sistema Winsor III o fase media. Este sistema contiene una nueva fase media, termodinámicamente estable que contiene todo el surfactante y una mezcla de agua y aceite; esta fase media coexiste con las fases en exceso de acuosa y orgánica. Finalmente, al adicionar una cantidad suficiente de surfactante, el sistema Winsor tipo III cambia a un sistema Winsor tipo IV, donde todo el surfactante, aceite y agua coexisten en una sola fase.

En la Figura 1 se muestra el comportamiento de fase para los sistemas formulados a una concentración de ACG de $0,5 \% \mathrm{~m} / \mathrm{v}$ variando la concentración de $\mathrm{NaCl}$, este grafico se caracteriza por presentar dos regiones con un comportamiento bifásico (WI y WII) que delimitan una zona trifásica (WIII). A bajas concentraciones de sal se obtienen sistemas del tipo Winsor I, ya que el surfactante se encuentra mayoritariamente en la fase acuosa, debido a que las interacciones relativas del anfifílo con esta fase predominan sobre las interacciones con la fase orgánica. Mientras que a concentraciones más elevadas de sal se obtienen sistemas del tipo Winsor II, en este caso el anfifílo tiende a solubilizarse en la fase orgánica, debido a que las interacciones de este con el hidrocarburo son mayores.

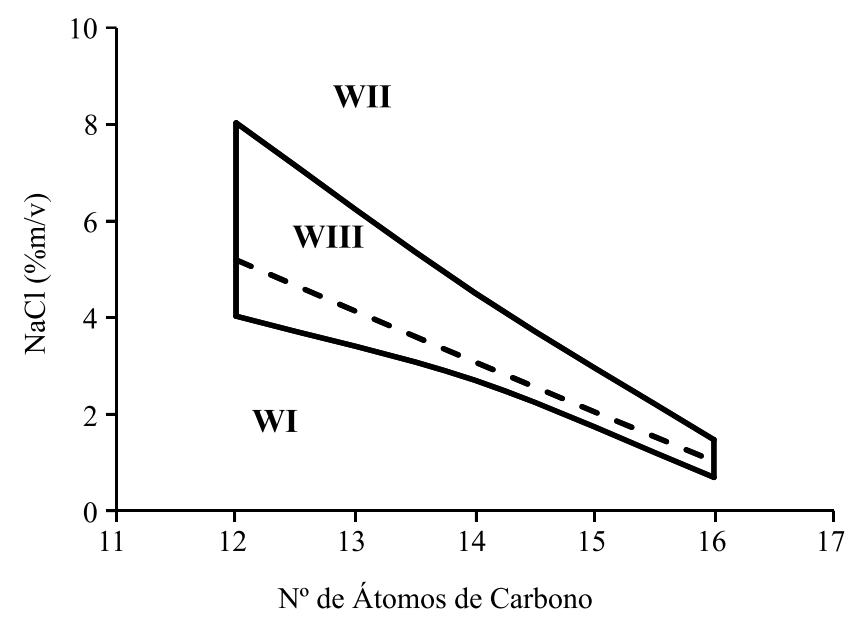

Figura 1. Comportamiento de fases para sistemas ACG/n-heptano/agua/n-butanol a una concentración de $0,5 \% \mathrm{~m} / \mathrm{v}$ de $\mathrm{NaCl}$

La línea punteada indica la concentración de electrolito donde se encuentran los sistemas tendientes a formulación óptima, es decir donde se ha solubilizado igual cantidad de agua y de $n$-heptano. Está bien reportado ${ }^{10-12}$ que en los sistemas a formulación óptima la tensión interfacial pasa por un mínimo, el tipo de emulsión se invierte, la estabilidad y la viscosidad de la emulsión son mínimas. Adicionalmente, se puede notar que la modificación en forma creciente de la variable de formulación en estudio (salinidad), produjo una transición en el comportamiento de fase de tipo $\mathrm{WI} \rightarrow \mathrm{WIII} \rightarrow$ WII.

La determinación de los sistemas tendientes a formulación óptima se realizó a través de las curvas del parámetro de solubilización (PS) (Ecuación 2), las cuales mostraron un comportamiento polinómico $\left(\mathrm{Y}=\mathrm{ax}^{2} \pm \mathrm{bx} \pm \mathrm{c}\right.$ ); por tanto, igualando la ecuación correspondiente al parámetro de solubilización en el hidrocarburo con la ecuación correspondiente al parámetro de solubilización en el agua se obtiene el valor de concentración óptima de electrolito (Figura 2). La determinación del sistema optimo se determina de forma visual por la transición de fase o correlacionando las curvas del parámetro de solubilización, ambos métodos son apropiados ya que presentan una buena correlación lineal.

\section{Estudio del coeficiente de reparto de ACG en sistemas Winsor III tendiente a formulación óptima}

Todas las variables de formulación son susceptibles de incrementar o disminuir la afinidad del surfactante tanto por la fase orgánica como por la fase acuosa, alterando de esta forma el valor de la relación $[\mathrm{ACG}]_{\mathrm{O}} /[\mathrm{ACG}]_{\mathrm{A}}$ y por lo tanto del coeficiente de reparto. Los resultados obtenidos mostraron que al incrementar la cadena hidrocarbonada del anfifílo aumenta el coeficiente de reparto de este; puesto que en la fase orgánica en exceso se distribuyó una mayor concentración de ácido con respecto a la distribuida en la fase acuosa como se 


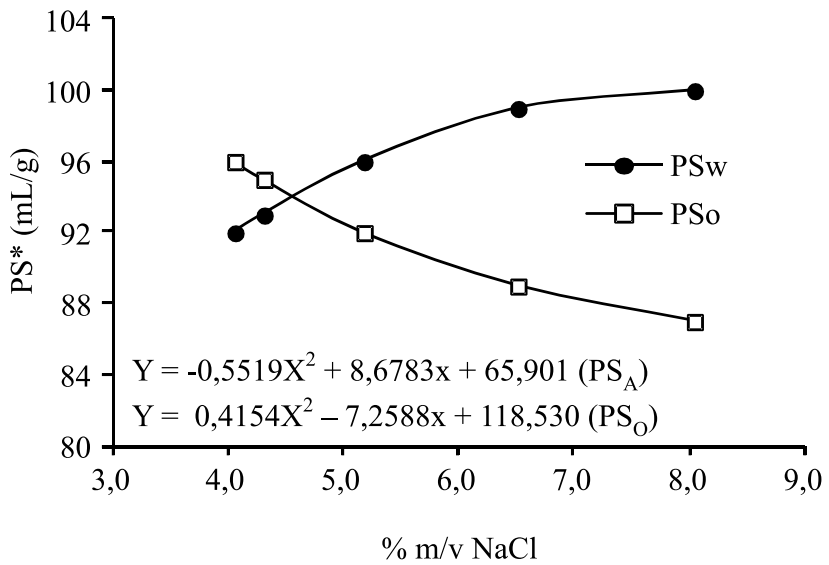

Figura 2. Parámetro de solubilización del sistema ácido dodecanóico a 0,5\% $\mathrm{m} / \mathrm{v}$ en función de la concentración de $\mathrm{NaCl}$

observa en la Figura 3. Efectivamente, la concentración del ácido en la fase acuosa estuvo limitada a su solubilidad en agua o la CMC. El coeficiente de reparto obtenido para los ácidos C12 y C14 indica que estos se distribuyen mayoritariamente en la fase acuosa, este comportamiento se debe a, que estos ácidos por poseer un menor número de átomos de carbono tienden a ser más hidrofílicos ofreciendo por lo tanto, mayor afinidad hacia la fase acuosa, de hecho los sistemas formulados con estos ácidos requieren una mayor concentración de electrolitos para obtener la transición de fase WI WIII WII.

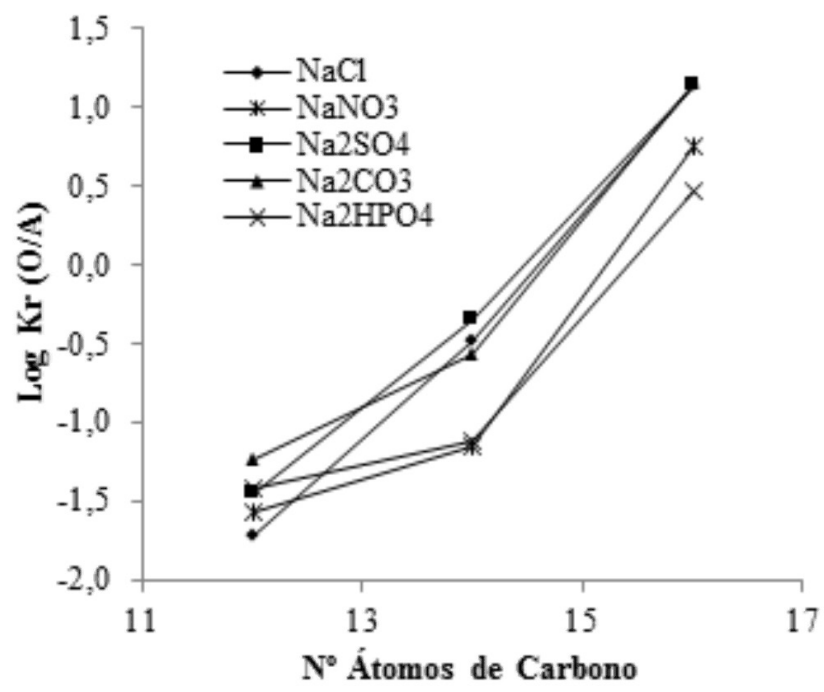

Figura 3. Coeficiente de reparto de los diferentes ACG a una concentración de $0,5 \% \mathrm{~m} / \mathrm{v}$ y los diferentes electrolitos estudiados

Para el caso del ácido C16 se observa que este por poseer un mayor número de átomos de carbono tiende a ser más lipofílico aumentando así su afinidad por la fase orgánica, este comportamiento se observó para todos los electrolitos estudiados, sin embargo para el caso del $\mathrm{NaNO}_{3}$ y del $\mathrm{Na}_{2} \mathrm{HPO}_{4}$ se evidenció que el reparto es inferior con respecto al resto de los electrolitos, esto puede ser debido a que el anión $\mathrm{NO}_{3}{ }^{-}$es considerado como un anión caotrópico, ${ }^{13}$ por lo tanto posee un efecto estabilizante o efecto salting in. Si embargo, el anión $\mathrm{HPO}_{4}{ }^{=}$no produce un marcado efecto salting out, esto puede ser atribuido a que este es el anión que posee el radio iónico más grande $(5,24 \AA)$, consecuentemente posee el radio de hidratación más pequeño $(0,0364 \AA)$ por lo tanto este producirá un menor efecto de desolvatación de la parte polar del anfifílo ( $\mathrm{RCOO}^{-}$), permitiendo que los iones carboxilato permanezcan en solución acuosa, estabilizados por los puentes de hidrógeno con las moléculas de agua o por la formación de dímeros entre moléculas de carboxilato/ácido caboxílico. ${ }^{15}$

El coeficiente de reparto se ve afectado por los electrolitos añadidos, ya que estos modificaran la CMC, las propiedades de los agregados, el tamaño y la forma de estos y la estabilidad de las fases. Por otro lado, la transferencia de una molécula de surfactante de la fase acuosa a la fase orgánica produce un cambio de energía libre de Gibbs que se puede calcular por el valor del coeficiente de reparto $\mathrm{K}_{\mathrm{R}}$ (Ecuación 4) siempre y cuando los coeficientes de actividad sean unitarios, lo que se cumple en general en ausencia de micelas como en el caso de las fases en exceso de un sistema Winsor III. Este parámetro fue posible determinarlo debido a que las concentraciones de ACG en las fases en exceso son suficientemente bajas, por lo tanto los valores obtenidos de $\mathrm{K}_{\mathrm{R}}$ para cada sistema se pueden relacionar con la energía libre de transferencia $\left(\Delta \mathrm{G}_{\mathrm{t}}\right)$ según la siguiente ecuación:

$$
\Delta G_{O \rightarrow A}=\mu^{*}{ }_{O}-\mu_{A}^{*}=R * T * \operatorname{Ln} K_{R}
$$

En el caso de los sistemas agua/hidrocarburo esta energía corresponde a la transferencia de $1 \mathrm{~mol}$ de ACG desde la fase orgánica hacia la fase acuosa, es decir, la energía libre de transferencia $\left(\Delta \mathrm{G}_{\mathrm{O} \rightarrow \mathrm{A}}\right)$ (Tabla 1). En la mayoría de los casos los valores de $\Delta \mathrm{G}_{\mathrm{O} \rightarrow \mathrm{A}}$ son negativos lo que indica que la transferencia de un mol de ACG hacia la fase acuosa es favorable; cabe destacar que a medida que se incrementa el número de átomos de carbono este proceso de transferencia se hace menos espontaneo; para el caso del C16 se obtienen valores de $\Delta \mathrm{G}_{\mathrm{O} \rightarrow \mathrm{A}}$ positivos lo que indica la resistencia a la transferencia de este ácido del $n$-heptano hacia el agua, para el caso de los sistemas de $\mathrm{C} 16$ con $\mathrm{NaNO}_{3}$ y $\mathrm{Na}_{2} \mathrm{HPO}_{4}$ se obtuvieron los menores valores de $\Delta \mathrm{G}_{\mathrm{O} \rightarrow \mathrm{A}}$, lo que indica que en estos sistemas la presencia de estos electrolitos favorece el proceso de transferencia de este ácido hacia la fase acuosa; estos valores concuerdan con los obtenidos para el coeficiente de reparto.

Tabla 1. Energía libre de transferencia $\left(\Delta \mathrm{G}_{\mathrm{O} \rightarrow \mathrm{A}}\right)$ para los sistemas con los diferentes electrolitos estudiados $(0,5 \% \mathrm{~m} / \mathrm{v})$

\begin{tabular}{cccccc}
\hline \multirow{2}{*}{$\begin{array}{c}\mathrm{N}^{\circ} \text { de } \\
\text { átomos de } \\
\text { carbonos }\end{array}$} & $\mathrm{NaCl}$ & $\mathrm{NaNO}_{3}$ & $\mathrm{Na}_{2} \mathrm{SO}_{4}$ & $\mathrm{Na}_{2} \mathrm{CO}_{3}$ & $\mathrm{Na}_{2} \mathrm{HPO}_{4}$ \\
\hline $\mathrm{C} 12$ & $-9,76$ & $-8,91$ & $-8,24$ & $-7,05$ & $-8,07$ \\
$\mathrm{C} 14$ & $-2,73$ & $-6,60$ & $-1,95$ & $-3,23$ & $-6,37$ \\
$\mathrm{C} 16$ & 6,53 & 4,31 & 6,53 & 6,54 & 2,72 \\
\hline
\end{tabular}

\section{CONCLUSIONES}

La variación de la concentración y naturaleza de electrolitos en los sistemas ACG/agua/n-heptano/n-butanol produjo transiciones de fase del tipo $\mathrm{WI} \rightarrow \mathrm{WIII} \rightarrow \mathrm{WII}$; así mismo la concentración del electrolito requerida para obtener los sistemas tendientes a formulación óptima varió inversamente proporcional con la longitud de la cadena del ácido. Los resultados mostraron que al incrementar la cadena hidrocarbonada del ácido aumenta el coeficiente de reparto del mismo. La medida del coeficiente de reparto de los ACG permitió obtener experimentalmente información termodinámica del proceso de transferencia del ácido del medio orgánico al medio acuoso el cual se ve afectado por el efecto hidrofóbico en torno a la cadena alquílica del ácido produciendo una reducción entálpica y entrópica.

\section{AGRADECIMIENTOS}

Al CONDES-LUZ de la Universidad del Zulia (CC-0423-11 y CC-0422-11), al FONACIT (PEI - 201 1001344) y FUNDACITEZulia por el financiamiento recibido. 


\section{REFERENCIAS}

1. Márquez, G.; Martín, J. J.; Alejandre, F. J.; Fortes, J. C.; Prat, F.; Dávila, J. M.; Mater. Construcc. 2009, 59, 79.

2. Filippo, C.; Pasquale, S.; Pier, L.: J. Colloid Interface Sci. 2010, 345, 561.

3. Liu, F.; Wu Wang, Z.; Chin. Chem. Lett. 2010, 21, 105.

4. Iglauer, S.; Wu, Y.; Shuler, P.; Tang, Y.; Goddard, W.; J. Petrol. Sci. Eng. 2010, 71, 23.

5. Bravo, B.; Sánchez, J.; Chávez, G.; Cáceres, A.; Ysambertt, F.; Márquez, N.; Ciencia 2007, 15, 270.

6. Xu. Q.; Nakajima, M.; Ichikawa, S.; Nakamura, N.; Roy, P.; Okadome, H.; Shiina, T.; J. Colloid Interface Sci. 2009, 332, 208.

7. Li, Y.; Ye, R.; Mu, R.; J. Surfact. Deterg. 2009, 12, 31.
8. Xenakis, A.; Papadimitriou, V.; Sotiroudis, T. G.; Curr. Opin. Colloid Interface Sci. 2010, 15, 55.

9. Do, L. D.; Withayyapayanon, A.; Harwell, J. H.; Sabatini, D. A.; J. Surfact. Deterg. 2008, 12, 91 .

10. Bravo, B.; Sánchez, J.; Cáceres, A.; Chávez, G.; Ysambertt, F.; Márquez, N.; Jaimes, M.; Briceño, M.; Salager, J. L.; J. Surfact. Deterg. 2008, 11, 13.

11. Salabat, A.; Alinoori, M. ; J. Calphad. 2008, 32, 611.

12. Misra, P. K.; Mishra, H. P.; Dash, U.; Mandal, A. B.; J. Colloid Interface Sci. 2009, 333, 590 .

13. Pegram, L. M.; Record, M. T.; Chem. Phys. Lett. 2008, 467, 1.

14. Winsor, P.; Solvent properties of amphiphilic compounds, Butterworths Scientific Publications: London, 1954, p. 1396-1415.

15. Long, B.; Wang, Y.; Yang, Z.; J. Chem. Thermodynamics 2008, 40, 1565. 